\title{
BMJ Open Study protocol for the international Systemic Lupus Erythematosus Prospective Observational Cohort Study (SPOCS): understanding lupus and the role of type I interferon gene signature
}

\author{
Edward R Hammond, ${ }^{1}$ Raj Tummala, ${ }^{2}$ Anna Berglind,${ }^{3}$ Farhat Syed,${ }^{4}$ Xia Wang, ${ }^{5}$ \\ Barnabas Desta, ${ }^{6}$ Henk $\mathrm{Nab}^{7}$
}

To cite: Hammond ER,

Tummala R, Berglind A, et al. Study protocol for the international Systemic Lupus Erythematosus Prospective Observational Cohort Study (SPOCS): understanding lupus and the role of type I interferon gene signature. BMJ Open 2020;10:e036563. doi:10.1136/ bmjopen-2019-036563

- Prepublication history and additional material for this paper are available online. To view these files, please visit the journal online (http://dx.doi org/10.1136/bmjopen-2019036563).

Received 19 December 2019 Revised 23 April 2020 Accepted 03 June 2020

Check for updates

(c) Author(s) (or their employer(s)) 2020. Re-use permitted under CC BY-NC. No commercial re-use. See rights and permissions. Published by BMJ.

For numbered affiliations see end of article.

Correspondence to

Dr Raj Tummala;

raj.tummala@astrazeneca.com

\section{ABSTRACT}

Introduction The Systemic Lupus Erythematosus (SLE) Prospective Observational Cohort Study (SPOCS) aims to describe the disease course of SLE and its association with type I interferon gene signature (IFNGS) status. Methods and analysis SPOCS is an international, multicentre, prospective, observational cohort study designed to follow patients through biannual study visits during a 3-year observation period. Patients $\geq 18$ years old with a physician diagnosis that meets the American College of Rheumatology or Systemic Lupus International Collaborating Clinics SLE classification criteria will be included. SPOCS will comprehensively analyse clinical features, disease progression and treatment, SLE outcomes, health status assessments and quality of life, and healthcare resource utilisation of patients with moderate to severe SLE. A four-gene test will be used to measure IFNGS status; scores will be compared with a pre-established cut-off. Patients will be stratified by low or high IFNGS expression levels. Enrolment began in June 2017, and study completion is expected in 2022. The total number of anticipated patients was initially planned for 1500 patients and was amended to 900 patients owing to slow accrual of eligible patients.

Ethics and dissemination The ethics committee/ institutional review board/independent ethics committee at each study site approved the SPOCS protocol prior to study initiation (protocol number: D3461R00001, version 3.0, 26 June 2019). Study findings will be disseminated through peer-reviewed publications and presentations at scientific meetings.

Trial registration number NCT03189875.

\section{INTRODUCTION}

Systemic lupus erythematosus (SLE) is a chronic autoimmune disorder with heterogeneous manifestations affecting various systems in the body. ${ }^{12}$ The pathophysiology of SLE involves immune activation, autoantibody production and deposition of immune complexes in various organ systems. This
Strengths and limitations of this study

- This study will provide comprehensive, longitudinal data, including biannual type I interferon gene signature (IFNGS) measurements for patients with moderate and severe systemic lupus erythematosus (SLE), rendering SLE Prospective Observational Cohort Study (SPOCS) data unique from current data available to existing SLE registries.

- Findings from SPOCS will improve understanding of the burden of SLE in the general population of patients with moderate to severe disease, including relating type I IFNGS to SLE disease course, as well as potentially showing gene signature differences between populations.

- Through collaboration with existing SLE registries and academic centres, SPOCS uses established patient recruitment methods, allowing efficient access to large numbers of patients.

- The observational design of SPOCS provides generalisable data that can be used in comparative effectiveness research and to contextualise findings from controlled trials.

- There is a possibility of missing data, especially during the follow-up period, owing to the observational study design; however, site staff will be trained on data collection methods and patients will receive reminders for appointments to reduce possible missing information.

inflammatory process may lead to organ damage. ${ }^{3}$

Patients with SLE present with diverse symptoms, including symptoms associated with the skin, kidney, joints, cardiovascular system and central nervous system. ${ }^{12}$ As a consequence, SLE presents as a major burden both in terms of decreased quality of life and increased economic costs. ${ }^{4-6}$ SLE is associated with significant impairment in patients' ability to work and adversely affects 
normal life activities and mental health. ${ }^{5}$ The SLE disease course is associated with high direct and indirect healthcare costs. ${ }^{5-7}$

Mainstay treatments for SLE, such as corticosteroids and immunosuppressants, may be associated with poor tolerability and substantial adverse events. ${ }^{89}$ Progress on novel therapies has been limited; belimumab, approved in 2011, has been the only new therapy for SLE approved in the past 50 years. ${ }^{510}$ Additionally, based on published guidelines, rituximab is considered an off-label treatment option for patients with lupus nephritis who do not respond to first-line therapy ${ }^{11}{ }^{12}$ and has been used as an off-label treatment in patients with SLE. ${ }^{13}$ Because SLE continues to present high disease burden, additional treatments are needed.

There is increasing evidence regarding the role of type I interferon (IFN) in the pathogenesis and disease course of SLE ${ }^{1415}$ and sequela associated with the type I IFN pathway, such as neuropsychiatric lupus and lupus nephritis. ${ }^{16} 17$ For patients with SLE, elevated type I IFN concentrations and overexpression of type I IFN-inducible gene signature (IFNGS) have been associated with increased SLE disease activity as measured by the Systemic Lupus Erythematosus Disease Activity Index (SLEDAI) score and higher anti-double-stranded DNA (anti-dsDNA) concentrations. ${ }^{14}{ }^{18-21}$ IFNGS is not measured through a genetic test, and measuring IFN mRNA gene signatures of individual patients has been found to be a more sensitive method for detecting IFN pathway activation than monitoring IFN protein concentrations. ${ }^{22}$ Several drugs that target the type I IFN pathway are currently being developed for the treatment of SLE, including anifrolumab, a fully human immunoglobulin G1א (IgG1א) monoclonal antibody that targets the IFN- $\alpha$ receptor (IFNAR1) and blocks IFNAR1-dependent signalling, currently in phase III clinical development (NCT02446899 and NCT02446912). ${ }^{23} 24$

The aims of the SLE Prospective Observational Cohort Study (SPOCS) are to (1) describe the patient journey regarding clinical outcomes over time for patients with moderate to severe SLE, (2) examine the prevalence of patients with high and low type I IFNGS in a real-world setting by geography, and (3) understand how type I IFNGS in patients with SLE may be associated with disease activity and clinical outcomes over time. Herein, we describe the methods and procedures used in the ongoing SPOCS study.

\section{METHODS AND ANALYSIS \\ Study design}

SPOCS is an international, multicentre, prospective, observational cohort study of patients with moderate to severe SLE that includes 2 years of enrolment plus a maximum of 3 years of longitudinal follow-up with biannual study visits for each patient (figure 1). This study aims to describe the patient journey in terms of clinical features, disease activity and progression, treatment patterns, clinical outcomes, patient-reported health outcomes (PRO) and healthcare resource utilisation in patients with moderate to severe SLE. To address the key goal of understanding the role of IFN in SLE, type I IFNGS will be measured at visits throughout the study, and analysis of patient outcomes will be stratified by high versus low IFNGS.

SPOCS will enrol study participants from North America (Canada and USA), Europe (France, Germany, Italy, Spain and UK) and Australia. All patients will be observed until completion of 3 years of follow-up, loss to follow-up or death, whichever occurs first.

Because SPOCS is an observational study, there will be no study treatment or control groups, and no restriction on types of treatments. Healthcare providers will administer treatment to patients only in accordance with routine care and will record patient treatments during each study visit. Biannual (every six months) follow-up visits are planned, during which blood samples will be obtained for type I IFNGS and the SPOCS biobank. Whenever possible, study visits will occur during regular on-site clinic visits consistent with routine care. Planned data collection will take place during and immediately following patient visits. All patient healthcare encounters

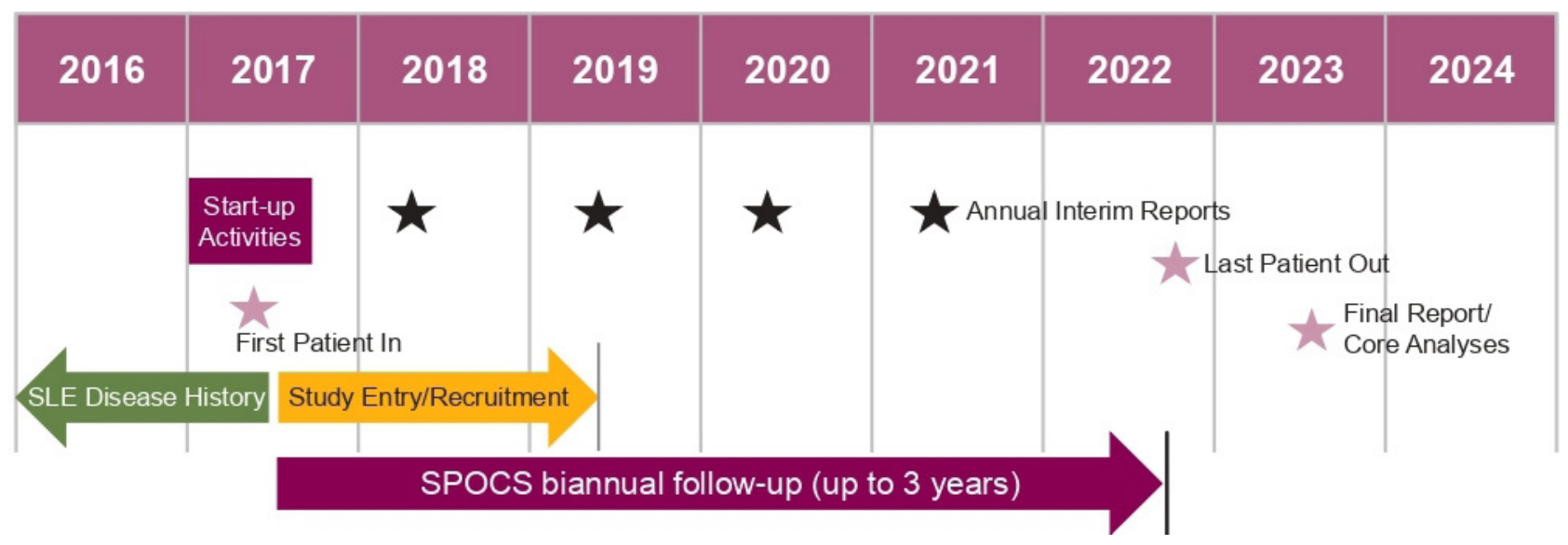

Figure 1 Study flow chart and plan, the SLE Prospective Observational Cohort Study (SPOCS). SLE, systemic lupus erythematosus. 
that occur as part of routine care after study entry and within 6 months prior to a subsequent study visit will be captured in the SPOCS database within a month of completing the study visit. At study sites, when possible, the study participant's encounter with the healthcare system may be entered into the database near the time of the healthcare encounter.

On-site and remote interim monitoring visits will be conducted by the clinical research associate and/or site manager to verify that the study is complying with the protocol and all applicable regulations and to confirm that all issues are resolved in a timely manner. For on-site monitoring, one visit per site will take place; for remote monitoring, there will be eight visits per site (occurring at approximately 32 -week intervals). Outbound contact by telephone to sites will also be made once per month or as needed to assess compliance with regulations, discuss patient enrolment, review data queries, and so on.

\section{Site and investigator selection}

Patients will be identified for potential enrolment in SPOCS through specialised SLE treatment and management centres, including academic centres, established SLE registries and independent specialist centres. SLE registries collaborating in this study include the Australian Lupus Registry and Biobank (ALRB), ${ }^{25}$ the Canadian Network for Improved Outcomes in SLE (CaNIOS), ${ }^{26}$ the French network of excellence in clinical research, continuation of the CRI (Rheumatism and Inflammation Club) and dedicated to IMIDs (Immune-Mediated Inflammatory Diseases) (CRI-IMIDIATE), ${ }^{27}$ the Spanish Society of Rheumatology SLE registry (RELESSER) ${ }^{28}$ and Systemic Lupus International Collaborating Clinics (SLICC) ${ }^{29}$ individual sites that agree to participate. To supplement patients identified and enrolled through existing partner SLE registries, physicians with expertise in treating adult patients with SLE will be identified and contacted to request participation of their practices as study sites, mainly in regions that are not covered by partner registries or where enrolment by partner registries is limited.

\section{Study participants}

Details of the complete inclusion and exclusion criteria are presented in box 1. Patients will be eligible to participate in SPOCS if they are 18 years or older, have a physicianconfirmed diagnosis of SLE by American College of Rheumatology or SLICC criteria, have a current or historic positive serology of antinuclear antibodies or dsDNA based on local/country laboratory reference range and have received a minimum of 6 months of systemic SLE treatment beyond non-steroidal anti-inflammatory drugs and analgesics. Patients will not be eligible to participate in the study if they are actively enrolled in interventional trials involving investigational agents at the time of enrolment or if they have severe lupus nephritis with a history of renal biopsy in the past year showing active class III or class IV \pm class $V$ lupus nephritis or a urine protein:creatinine ratio $>1 \mathrm{mg} / \mathrm{mg}$ based on random urine collection
Box 1 Inclusion/exclusion criteria for patient enrolment in SPOCS

Inclusion criteria

- Patients $\geq 18$ years old.

- Physician confirmation that patient meets $\mathrm{ACR}^{38}$ or SLICC ${ }^{38} 39$ SLE classification criteria.

- Current or previous positive serology of ANA or dsDNA.

- Minimum 6-month treatment duration for active SLE with systemic SLE treatment beyond NSAIDs and analgesics.

- Moderate to severe SLE; SLEDAI-2K criteria: modified SLEDAI-2K score $\geq 4$ points, defined as SLEDAI-2K assessment score without the inclusion of points attributable to any urine or laboratory results, including immunological measures and lupus headache; or SLEDAI$2 \mathrm{~K}$ score $\geq 6$ points.

- Patient and/or representative(s) who understands the requirements of the study and provides written informed consent.

Exclusion criteria

- Patients actively enrolled in interventional trials involving investigational agents.

- Patient with active severe lupus nephritis with a history of renal biopsy in the last year showing active class III or class IV \pm class V lupus nephritis and/or urine protein:creatinine ratio $>1 \mathrm{mg} / \mathrm{mg}$ based on random urine collection at baseline visit.

- Patients unable to complete study measures.

ACR, American College of Rheumatology; ANA, antinuclear antibodies; dsDNA, double-stranded DNA; NSAID, non-steroidal anti-inflammatory drug; SLE, systemic lupus erythematosus; SLEDAI-2K, Systemic Lupus Erythematosus Disease Activity Index 2000; SLICC, Systemic Lupus International Collaborating Clinics; SPOCS, SLE Prospective Observational Cohort Study.

at the baseline visit. Patients who develop severe, active lupus nephritis or severe central nervous system disease during participation in SPOCS may remain in the study. Patients enrolled in SPOCS who later enrol in interventional trials involving investigational agents will be permitted to remain in SPOCS. Enrolled patients can withdraw from participation at their own discretion, at any time and without prejudice to their subsequent medical treatment. Patients who withdraw prematurely will not be replaced.

Patient enrolment began in June 2017, and study completion is anticipated in 2022. The planned enrolment for SPOCS was initially 1500 patients, which was amended to 900 patients with an approximate distribution per participating country as follows: Australia, 80; Canada, 80; France, 80; Germany, 80; Italy, 80; Spain, 100; UK, 100; USA, 300. All eligible patients who are not recruited in SPOCS will be registered in a screening log, as allowed per local regulations, with basic characteristics (eg, age, SLE severity) and the reason for non-inclusion. Strategies to achieve adequate patient enrolment include site staff training, monthly site phone calls to review recruitment with site study team, enrolment logs to observe the site's effort with recruitment and address potential barriers, investigator teleconferences between sites to share experiences and best practices and study 


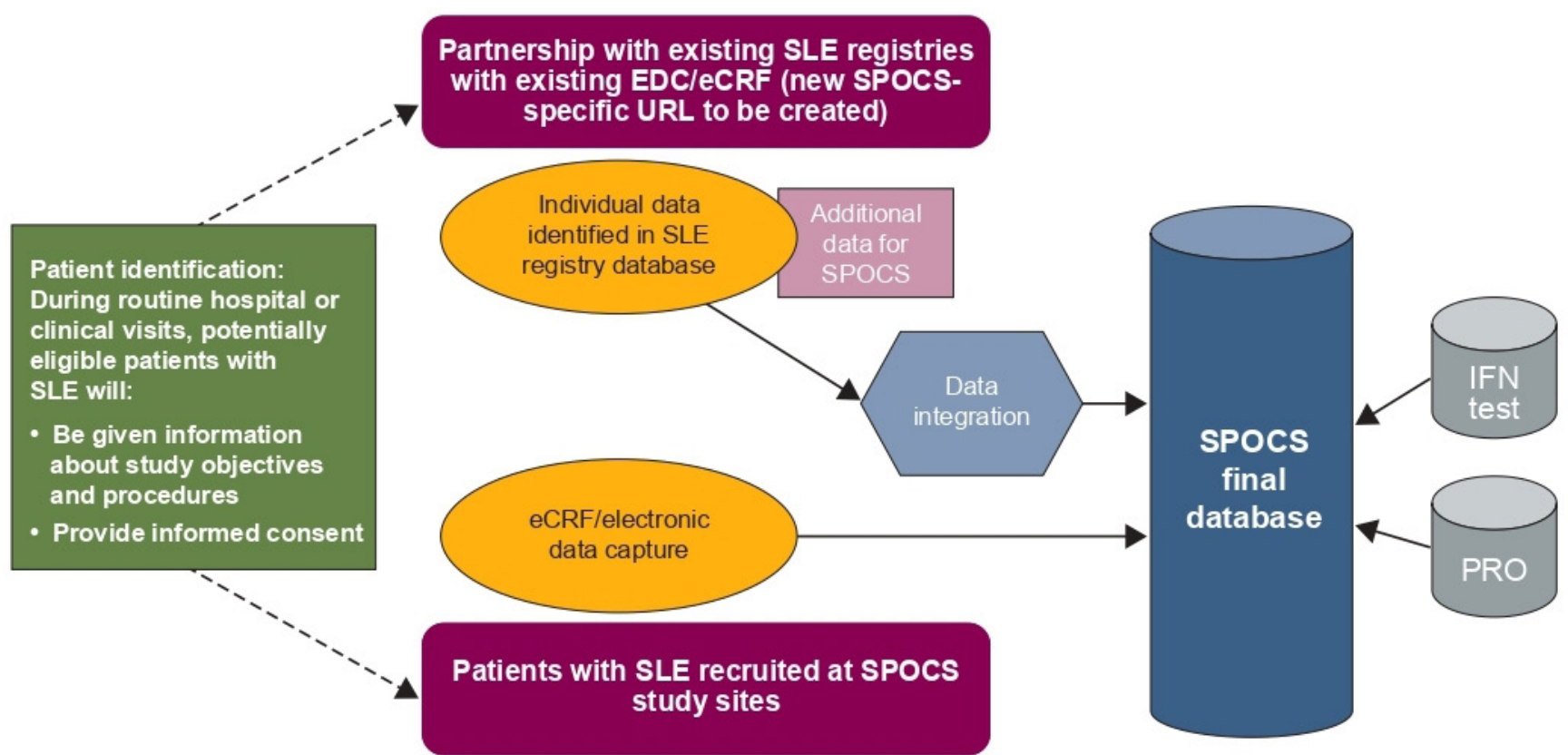

Figure 2 Patient recruitment and data collection, the SLE Prospective Observational Cohort Study (SPOCS). eCRF, electronic case report form; EDC, electronic data capture; IFN, interferon; PRO, patient-reported outcome; SLE, systemic lupus erythematosus.

newsletters to provide progress updates and raise awareness of recruitment achievements and/or challenges.

\section{Data collection}

Data for patients enrolled in SPOCS will be obtained through primary data collection methods, including SPOCS-specific electronic case report forms (eCRF) and PRO instruments, and collected routinely from existing SLE registry partners. Data collection will take place at study entry and every 6 months at patient follow-up visits. Each patient is expected to complete seven study visits over the planned 3-year follow-up period. Should any patient decide to withdraw from the study, a final assessment will be requested of the patient at the time of withdrawal. Relevant medical data from intervals between study visits will be captured at the subsequent study visit using medical records and questionnaires.

Approaches for data collection are summarised in figure 2. Deidentified data for patients obtained from an existing SLE registry (eg, ALRB) who sign an informed consent form will be captured by a SPOCS-specific website that contains all SPOCS variables created for the respective SLE registry. These deidentified data include age group, sex, SLE severity, years since SLE diagnosis and the reason non-enrolled patients did not participate. Data will be electronically transferred and integrated into the SPOCS database on an ongoing basis. When patients enrol through a study site or an existing SLE registry partner acting as an SPOCS site, full primary data will be collected via eCRFs and/or PROs through the participating site and entered into the SPOCS database. Testing for type I IFNGS will be conducted in a central lab, with results linked to the individual patient and integrated directly into the SPOCS database. Standard data quality assurance processes will be in place, and monitoring will be implemented. Site managers or clinical research associates will conduct on-site monitoring to ensure all data are entered and queries are resolved. Data will be reviewed on an ongoing basis to identify any outstanding data and query resolution issues. In addition, a close collaboration between AstraZeneca and associated sites will be fostered to ensure data quality is maintained at each study site.

\section{Type I IFNGS}

A blood sample for evaluating type I IFNGS will be obtained at each study visit. Whole blood will be collected in PAXgene Blood RNA tubes. RNA will be isolated from blood using a PAXgene Blood RNA kit, per manufacturer instructions. The type I IFNGS test will be conducted at a designated central laboratory using the QIAGEN therascreen IFN-inducible gene expression (IFIGx) Rotor-Gene Q (RGQ) reverse transcriptase PCR (RT-PCR) system. This expression system consists of collection tubes and RNA isolation kits described above, along with a therascreen IFIGx RGQ RT-PCR kit used with RGQ molecular diagnostic platform with IFIGx software. The IFIGx kit measures the expression of four type I IFN-inducible genes (IFI27, IFI44, IFI44L and RSAD2) relative to three housekeeping genes (ACTB, $18 S$ and GAPDH) and generates a qualitative diagnostic score of positive or negative. The result is expressed as a score that is compared with a pre-established delta Ct-based cut-off, in the trough of the bimodal distribution, and classifies patients into one of two groups representing low or high IFIGx. The analytical validation of the four-gene test as a measurement of IFIGx has been reported and is currently being used in two phase III studies of anifrolumab for the treatment of SLE (NCT02446899 and NCT02446912). ${ }^{30}$ Patients 
with SLE, who present heterogeneous disease activity and symptoms, are commonly stratified by low or high levels of IFIGx. ${ }^{2031}$

\section{Study outcomes}

SPOCS is designed to systematically describe the SLE disease course with respect to clinical features, disease progression and treatment, SLE outcomes, health status assessments through PROs and healthcare resource utilisation in a general population of patients with moderate to severe SLE. This study will evaluate cross-sectional and longitudinal associations of assessed parameters and outcomes for patients overall and by type I IFNGS status (box 2), potentially showing gene signature differences between populations. Additionally, the data obtained through SPOCS will allow evaluation of epidemiological and clinical concepts related to the natural history of SLE in subpopulations.

\section{Biobank and sample storage}

Biological samples will be collected by site personnel for analysis at a central laboratory (type I IFNGS) or storage in a central biospecimen repository (SPOCS biobank) for future analysis. Biological samples will be retained at a biobank for a maximum of 15 years following the finalisation of the clinical study report. Collected blood samples will be deidentified for storage, and the central repository will not contain identifying data.

\section{Sample size estimation}

A sample size of 1500 patients was initially planned based on cross-sectional precision estimates of proposed disease activity measures at study entry and was deemed adequate to effectively characterise the important endpoints and comparisons likely to occur during the study through various analytical techniques. However, this sample size was amended to 900 patients because of slow accrual of eligible patients. Study precision estimates were revised accordingly.

\section{Patient and public involvement}

Patients were not involved in the design of this study. However, learnings gained from patient perspectives about the burden of completing questionnaires and tests, which were gathered from clinical trial experience, were considered in the study design. Patients who consent to participate in the study will spend additional time during routine hospital visits to complete study questionnaires and additional blood tests for type I IFNGS.

\section{Statistical analysis}

Data obtained in SPOCS will be reported descriptively, overall and by type I IFNGS test status to address the objectives of the study through outcomes listed in box 2 . The prevalence of high or low type I IFNGS will be reported by geographic area. Planned analyses include a description of the study population and characterisation of clinical features, healthcare resource utilisation and clinical outcomes for patients with moderate to severe SLE, and

\section{Box 2 Data to be collected in SPOCS}

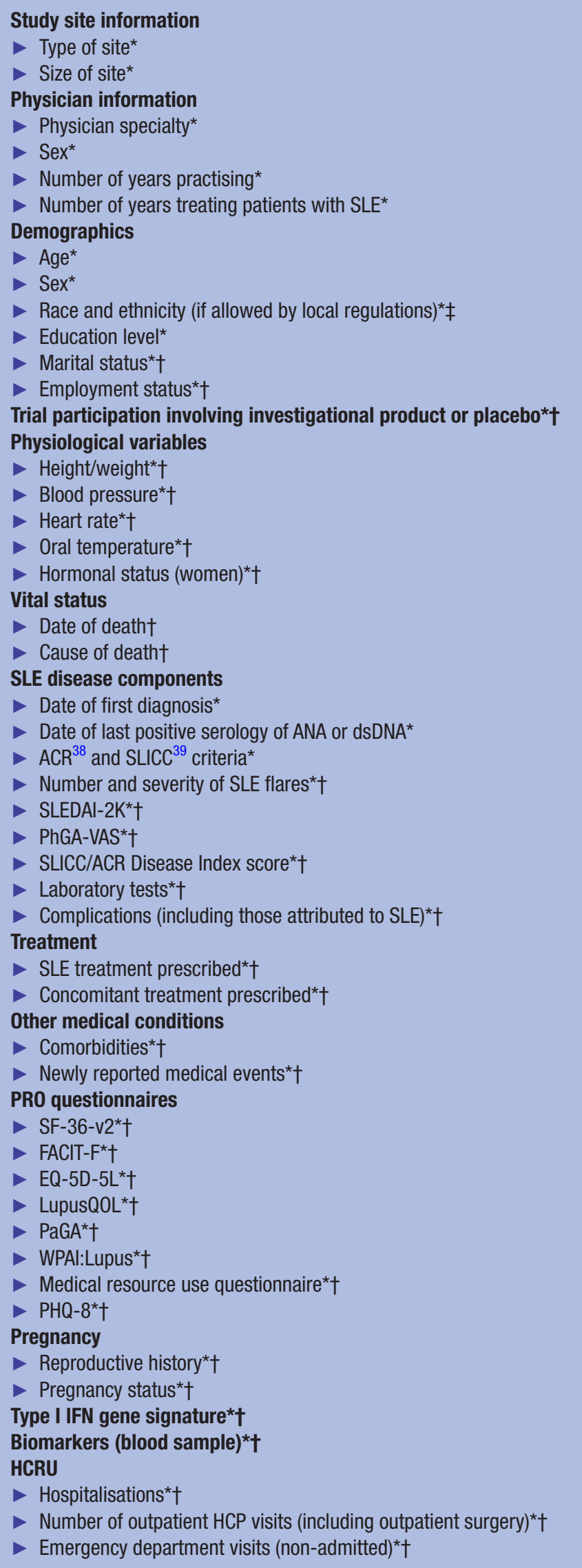

Continued 


\section{Box 2 Continued}

*Information collected at study entry.

†Information collected at biannual follow-up visits.

fLocal data protection laws in some countries where we have study sites that may preclude these data being collected.

ACR, American College of Rheumatology; ANA, antinuclear antibodies; dsDNA, double-stranded DNA; EQ-5D-5L, EuroQ0L 5 Dimensions 5 Levels; FACIT-F, Functional Assessment of Chronic IIIness TherapyFatigue; HCP, healthcare provider; HCRU, healthcare resource utilisation; IFN, interferon; LupusQ0L, Lupus Quality-of-Life instrument; PaGA, Patient's Global Assessment of disease activity; PhGA, Physician's Global Assessment of disease activity; PHQ-8, Personal Health Questionnaire depression scale; PR0, patient-reported outcome; SF36-v2, Short Form 36 Health Survey, version 2; SLE, systemic lupus erythematosus; SLEDAI-2K, Systemic Lupus Erythematosus Disease Activity Index 2000; SLICC, Systemic Lupus International Collaborating Clinics; SPOCS, SLE Prospective Observational Cohort Study; VAS, visual analogue scale; WPAl:Lupus, Work Productivity and Activity Impairment Questionnaire:Lupus.

distribution of the type I IFNGS (test high vs test low) and any potential associations with SLE disease activity and outcomes. A statistical analysis plan will detail calculations of derived variables and descriptive analyses of prespecified subgroup analyses. The following measures will be reported as appropriate: mean, $\mathrm{SD}$, median, quartiles $(\mathrm{Q} 1$ and $\mathrm{Q} 3)$, geometric mean, geometric $\mathrm{SD}$, minimum and maximum. Change from baseline (where baseline is defined as study entry) summaries will include $95 \%$ CIs. To support descriptive analyses, further analyses including multivariate analytical methods and nested case-control design approaches may be used. Missing data will be reported as a percentage for each measured variable in the study and as a separate category for descriptive analyses. Annual interim progress reports that provide an overview of the study status, including enrolment and follow-up, distribution of patients and disease characteristics, are planned throughout the course of the study. Because SPOCS is a prospective cohort study, opportunities to update the statistical analysis plan have been built within study operations, prior to database lock. The Project Team and Scientific Committee will determine and approve the exact structure of each interim statistical analysis plan.

\section{ETHICS AND DISSEMINATION}

The ethics committee/institutional review board (IRB)/ independent ethics committee (IEC) at each study site approved the SPOCS protocol prior to study initiation (protocol number: D3461R00001, version 3.0) (online supplementary material). Patients and/or representative(s) are given full and adequate information about the nature, purpose, and possible risks and benefits of the study, providing confirmation that they understand the study requirements as well as written informed consent. Patient informed consent forms, which incorporate relevant data protection and privacy legislation information, are reviewed with the patient by the recruiting physician and signed by patients and/or representative(s) prior to study entry. Consent is sought from patients to allow further storage of a blood sample within the SPOCS biobank for future biomarker testing. All collected data are stored in SPOCS databases, maintaining confidentiality in accordance with local law. Data collection and deidentification are done in accordance with European Medicines Agency Policy $0070 .^{32}$

SPOCS is being conducted in accordance with the principles of the Declaration of Helsinki and is consistent with the International Council for Harmonisation Good Clinical Practices, Good Pharmacoepidemiology Practice and applicable legislation according to the study classification in each country. SPOCS has been registered with ClinicalTrials.gov since 16 June 2017, and the protocol was updated to reflect changes in sample size on 26 June 2019. Changes to the protocol will be documented in written amendments and communicated to relevant parties, including updates to study information on ClinicalTrials.gov. Major amendments will be approved by the relevant regulatory authorities with submission or notification to the relevant IRB/ IEC for approval as required. Minor protocol amendments will be filed at each participating site and submitted to the relevant IRB/IEC or regulatory authorities where required by pertinent regulations. Any amendment that could have an impact on the patient's agreement to participate in the study requires the patient's informed consent prior to continuing in the study.

A final study report is planned within 12 months after completion of the third year of follow-up for the last patient. All reporting will be consistent with the Strengthening the Reporting of Observational Studies in Epidemiology initiative checklist for cohort studies. ${ }^{33}$ Results of the study will be disseminated through submission of peer-reviewed manuscripts. Interim and final results may also be disseminated through publication or presentation at scientific meetings ${ }^{34}$ with input from the study Scientific and Executive Committee.

\section{DISCUSSION}

The SPOCS study will provide a comprehensive, longitudinal data source that is currently unique for this patient population; these types of data and size of this data set will be unmatched by current data sets or SLE registries. The wealth of data will be used to address the objectives of SPOCS, which are to describe the disease course for patients with moderate to severe SLE, examine the prevalence of patients with high versus low type I IFNGS and understand how type I IFNGS may be associated with disease activity and clinical outcomes over time.

A benefit of observational research is the ability to provide generalisable data that can be used in comparative effectiveness research and to contextualise findings from controlled trials. ${ }^{35}$ SPOCS combines this strength with the recruitment of a large international cohort of patients with moderate to severe SLE to improve understanding of 
SLE natural history, disease characteristics and burden in terms of disease severity, pharmacoeconomic impact and patients' health-related quality of life. With its overall analysis of patient outcomes and subgroup analysis comparing patients with high and low type I IFNGS, this study seeks to improve our understanding of the role of type I IFNGS in the SLE disease course. Analysis of outcomes by country and geographic region further improves understanding of the clinical and economic burden of SLE, which is known to have regional variation in prevalence, disease severity and outcomes. ${ }^{36}{ }^{37}$ Through collaboration with existing registries and academic centres, SPOCS takes advantage of established patient recruitment methods from current SLE registry partners, allowing efficient access to large numbers of patients.

Because SPOCS is an observational study, potential limitations need to be considered, including the possibility for missing data especially during the follow-up period. To minimise missing information, site staff will be trained on data collection methods. SPOCS will follow patients over 3 years, and some patients may be lost during the follow-up period. To reduce the number of patients lost to follow-up, patients will receive reminders of upcoming appointments via phone calls or emails. When healthcare providers do not see patients for more than 6 months, they will follow-up by telephone or email. A further potential limitation is that the type I IFNGS test used in this study classifies patients as having low or high IFIGx but does not provide a quantitative measure of IFN gene expression. Therefore, it does not directly allow for quantitative analysis of IFN gene expression by changes in disease state. Future approaches to analyse the data obtained in SPOCS, including nested case-control studies, will permit us to further evaluate and improve understanding of the potentially time-varying nature of the IFNGS and how it relates to SLE disease activity.

Results from SPOCS will provide a collection of data that will improve our understanding of SLE disease activity and how it translates to long-term clinical outcomes for patients with moderate to severe SLE. These data may enable identification of subgroups of patients at risk for disease progression or certain disease outcomes. Such findings may further our understanding of reported associations between IFNs and outcomes such as neuropsychiatric lupus and lupus nephritis. ${ }^{16}{ }^{17}$ Data obtained from SPOCS regarding type I IFNGS may be useful in understanding SLE and other diseases in which type I IFN pathways play a significant role.

\footnotetext{
Author affiliations

${ }^{1}$ BioPharmaceuticals Medical, AstraZeneca, Gaithersburg, Maryland, USA

${ }^{2}$ Inflammation, Autoimmunity and Neuroscience, AstraZeneca, Gaithersburg, Maryland, USA

${ }^{3}$ BioPharmaceuticals, R\&D, AstraZeneca, Gothenburg, Sweden

${ }^{4}$ Precision Medicine, R\&D, AstraZeneca, Cambridge, UK

${ }^{5}$ Data Science and Al, R\&D, AstraZeneca, Gaithersburg, Maryland, USA

${ }^{6}$ Global Pricing and Market Access, AstraZeneca, Gaithersburg, Maryland, USA

${ }^{7}$ Inflammation and Autoimmunity, AstraZeneca, Cambridge, UK
}

Acknowledgements Editorial assistance was provided by Alan Saltzman, PhD, CMPP, of JK Associates, a member of the Fishawack Group of Companies, Conshohocken, PA, USA, and Kathleen Jenks-Grobben, PhD, of AstraZeneca, Gaithersburg, MD, USA.

Contributors All authors (ERH, RT, AB, FS, XW, BD and HN) were involved in the conception or design of the study, helped draft or revise the manuscript for critically important intellectual content, approved the final version of the submitted manuscript and agree to be accountable for the accuracy and integrity of all aspects of the work. ERH and $A B$ were responsible for development of the statistical analysis plan.

Funding This work was supported by AstraZeneca.

Competing interests $\mathrm{ERH}, \mathrm{RT}, \mathrm{AB}, \mathrm{BD}, \mathrm{FS}$ and $\mathrm{XW}$ are AstraZeneca employees. HN was an employee at AstraZeneca at the time of the study.

Patient and public involvement Patients and/or the public were not involved in the design, or conduct, or reporting, or dissemination plans of this research.

Patient consent for publication Not required.

Provenance and peer review Not commissioned; externally peer reviewed.

Open access This is an open access article distributed in accordance with the Creative Commons Attribution Non Commercial (CC BY-NC 4.0) license, which permits others to distribute, remix, adapt, build upon this work non-commercially, and license their derivative works on different terms, provided the original work is properly cited, appropriate credit is given, any changes made indicated, and the use is non-commercial. See: http://creativecommons.org/licenses/by-nc/4.0/.

\section{REFERENCES}

1 Dema B, Charles N. Advances in mechanisms of systemic lupus erythematosus. Discov Med 2014;17:247-55.

2 Liu Z, Davidson A. Taming lupus-a new understanding of pathogenesis is leading to clinical advances. Nat Med 2012;18:871-82.

3 Luo S, Wang Y, Zhao M, et al. The important roles of type I interferon and interferon-inducible genes in systemic lupus erythematosus. Int Immunopharmacol 2016;40:542-9.

4 Lateef A, Petri M. Unmet medical needs in systemic lupus erythematosus. Arthritis Res Ther 2012;14 Suppl 4:S4

5 Carter EE, Barr SG, Clarke AE. The global burden of SLE: prevalence, health disparities and socioeconomic impact. Nat Rev Rheumatol 2016;12:605-20.

6 Pons-Estel GJ, Alarcón GS, Scofield L, et al. Understanding the epidemiology and progression of systemic lupus erythematosus. Semin Arthritis Rheum 2010;39:257-68.

7 Hammond E, Murimi IB, Lin DH. Health care utilization and costs of systemic lupus erythematosus (SLE) in the United States: a systematic review. Ann Rheum Dis 2017;76.

8 Gladman DD, Urowitz MB, Rahman P, et al. Accrual of organ damage over time in patients with systemic lupus erythematosus. $J$ Rheumatol 2003;30:1955-9.

9 Kaul A, Gordon C, Crow MK, et al. Systemic lupus erythematosus. Nat Rev Dis Primers 2016;2:16039.

10 Lichtman El, Helfgott SM, Kriegel MA. Emerging therapies for systemic lupus erythematosus--focus on targeting interferon-alpha. Clin Immunol 2012;143:210-21.

11 Bertsias GK, Tektonidou M, Amoura Z, et al. Joint European League against rheumatism and European renal AssociationEuropean dialysis and transplant association (EULAR/ERA-EDTA) recommendations for the management of adult and paediatric lupus nephritis. Ann Rheum Dis 2012;71:1771-82.

12 Hahn BH, McMahon MA, Wilkinson A, et al. American College of rheumatology guidelines for screening, treatment, and management of lupus nephritis. Arthritis Care Res 2012;64:797-808.

13 Aguiar R, Araújo C, Martins-Coelho G, et al. Use of rituximab in systemic lupus erythematosus: a single center experience over 14 years. Arthritis Care Res 2017;69:257-62.

14 Dall'era MC, Cardarelli PM, Preston BT, et al. Type I interferon correlates with serological and clinical manifestations of SLE. Ann Rheum Dis 2005;64:1692-7.

15 Bennett L, Palucka AK, Arce E, et al. Interferon and granulopoiesis signatures in systemic lupus erythematosus blood. J Exp Med 2003;197:711-23.

16 Bialas AR, Presumey J, Das A, et al. Microglia-Dependent synapse loss in type I interferon-mediated lupus. Nature 2017;546:539-43.

17 Castellano G, Cafiero C, Divella C, et al. Local synthesis of interferonalpha in lupus nephritis is associated with type I interferons signature 
and LMP7 induction in renal tubular epithelial cells. Arthritis Res Ther 2015;17:72.

18 Higgs BW, Liu Z, White B, et al. Patients with systemic lupus erythematosus, myositis, rheumatoid arthritis and scleroderma share activation of a common type I interferon pathway. Ann Rheum Dis 2011;70:2029-36.

19 Furie R, Khamashta M, Merrill JT, et al. Anifrolumab, an AntiInterferon- $\alpha$ receptor monoclonal antibody, in moderate-to-severe systemic lupus erythematosus. Arthritis Rheumatol 2017;69:376-86.

20 Chiche L, Jourde-Chiche N, Whalen E, et al. Modular transcriptional repertoire analyses of adults with systemic lupus erythematosus reveal distinct type I and type II interferon signatures. Arthritis Rheumatol 2014;66:1583-95.

21 Banchereau R, Hong S, Cantarel B, et al. Personalized immunomonitoring uncovers molecular networks that stratify lupus patients. Cell 2016;165:551-65.

22 Baechler EC, Batliwalla FM, Karypis G, et al. Interferon-Inducible gene expression signature in peripheral blood cells of patients with severe lupus. Proc Natl Acad Sci U S A 2003;100:2610-5.

23 clinicaltrials.gov. Efficacy and safety of anifrolumab compared to placebo in adult subjects with active systemic lupus erythematosus. Available: https://clinicaltrials.gov/ct2/show/NCT02446899?term= anifrolumab\&phase $=2 \&$ rank $=1$ [Accessed 5 Sep 2019].

24 clinicaltrials.gov. Efficacy and safety of two doses of anifrolumab compared to placebo in adult subjects with active systemic lupus erythematosus. Available: https://clinicaltrials.gov/ct2/show/ NCT02446912?term=anifrolumab\&phase $=2 \&$ rank $=3$ [Accessed 5 Sep 2019].

25 Vincent FB, Bourke P, Morand EF, et al. Focus on systemic lupus erythematosus in Indigenous Australians: towards a better understanding of autoimmune diseases. Intern Med $J$ 2013;43:227-34

26 Peschken CA, Katz SJ, Silverman E, et al. The 1000 Canadian faces of lupus: determinants of disease outcome in a large multiethnic cohort. J Rheumatol 2009;36:1200-8.

27 Imidiate. The word of the Steering Committee. Available: http://www. imidiate.org/en/objectives [Accessed 5 Sep 2019].

28 Rúa-Figueroa I, López-Longo FJ, Calvo-Alén J, et al. National Registry of patients with systemic lupus erythematosus of the Spanish Society of rheumatology: objectives and methodology. Reumatol Clin 2014;10:17-24.

29 Isenberg DA, Ramsey-Goldman R, Gladman D, et al. The Systemic Lupus International Collaborating Clinics (SLICC) group - it was 20 years ago today. Lupus 2011;20:1426-32.
30 Brohawn PK, Higgs BW, Patel S, et al. AB1212 analytical validation of an interferon-inducible gene expression kit as a potential diagnostic test for anifrolumab. Ann Rheum Dis 2018;77:1704-5.

31 Kennedy WP, Maciuca R, Wolslegel K, et al. Association of the interferon signature metric with serological disease manifestations but not global activity scores in multiple cohorts of patients with SLE. Lupus Sci Med 2015;2:e000080.

32 European Medicines Agency. External guidance on the implementation of the European medicines Agency policy on the publication of clinical data for medicinal products for human use. Available: https://www.ema.europa.eu/en/documents/regulatoryprocedural-guideline/external-guidance-implementation-europeanmedicines-agency-policy-publication-clinical-data_en.pdf [Accessed 5 Sep 2019].

33 von Elm E, Altman DG, Egger M, et al. The strengthening the reporting of observational studies in epidemiology (STROBE) statement: guidelines for reporting observational studies. Int J Surg 2014;12:1495-9.

34 Hammond ER, Aringer M, Arnaud L. Systemic lupus erythematosus disease characteristics associated with the type I interferon gene signature: baseline data of the SLE prospective observational cohort study (SPOCS). oral presentation OP0128 at: European Congress of rheumatology (EULAR); June 12-15. Madrid, Spain, 2019.

35 Hess LM, Kern DM, Carter GC, et al. Real-World treatment sequences and outcomes among patients with non-small cell lung cancer (RESOUNDS) in the United States: study protocol. JMIR Res Protoc 2017;6:e195.

36 Danchenko N, Satia JA, Anthony MS. Epidemiology of systemic lupus erythematosus: a comparison of worldwide disease burden. Lupus 2006;15:308-18.

37 Jakes RW, Bae S-C, Louthrenoo W, et al. Systematic review of the epidemiology of systemic lupus erythematosus in the Asia-Pacific region: prevalence, incidence, clinical features, and mortality. Arthritis Care Res 2012;64:159-68.

38 American College of Rheumatology. 1997 update of the 1982 American College of rheumatology revised criteria for classification of systemic lupus erythematosus, 1997. Available: https://www. rheumatology.org/Portals/0/Files/1997\%20Update\%20of\%201982\% 20Revised.pdf [Accessed 11 Oct 2017].

39 Petri M, Orbai A-M, Alarcón GS, et al. Derivation and validation of the systemic lupus international collaborating clinics classification criteria for systemic lupus erythematosus. Arthritis Rheum 2012;64:2677-86. 